\title{
Multipartite quantum correlation and entanglement in four-qubit pure states
}

\author{
Yan-Kui Bai, Dong Yang, and Z. D. Wang* \\ Department of Physics and Center of Theoretical and Computational Physics, University of Hong Kong, Pokfulam Road, \\ Hong Kong, China
}

(Received 20 March 2007; published 31 August 2007)

\begin{abstract}
Based on the quantitative complementarity relations, we analyze thoroughly the properties of multipartite quantum correlation and entanglement in four-qubit pure states. It is found that, unlike the three-qubit case, the single residual correlation and the genuine correlations of three and four qubits are unable to quantify entanglement appropriately. More interestingly, from our qualitative and numerical analysis, it is conjectured that the sum of all residual correlations is a good quantity for characterizing the multipartite entanglement in the system.
\end{abstract}

DOI: 10.1103/PhysRevA.76.022336

\section{INTRODUCTION}

Entanglement has been a vital physical resource for quantum information processing, such as quantum communication $[1,2]$ and quantum computation [3-5]. Therefore, the characterization of entanglement for a given quantum state is a fundamental problem. Bipartite entanglement is well understood in many aspects [6-9]. Especially, for two qubits, its mixed state entanglement can be characterized with the help of the so-called concurrence [10]. However, in multipartite cases, the quantification of entanglement is very complicated and challenging.

A fundamental property of multipartite entangled state is that entanglement is monogamous. In a three-qubit composite system $\rho_{A B C}$, the monogamy means that there is a trade off between the amount of entanglement that is shared by $\rho_{A B}$ and $\rho_{A C}$, respectively. For the pure state $|\Psi\rangle_{A B C}$, Coffman, Kundu, and Wootters proved the inequality $C_{A B}^{2}+C_{A C}^{2}$ $\leqslant \tau_{A\left(R_{A}\right)}$ [11], where the square of the concurrence $C_{i j}$ quantifies the entanglement of subsystem $\rho_{i j}$ and the linear entropy $\tau_{A\left(R_{A}\right)}$ measures the pure state entanglement between qubit $A$ and remaining qubits $B C$. Particularly, the residual quantum correlation in the above equation, i.e., the three tangle

$$
\tau\left(\Psi_{A B C}\right)=\tau_{A\left(R_{A}\right)}-C_{A B}^{2}-C_{A C}^{2},
$$

was proven to be a good measure for genuine three-qubit entanglement $[11,12]$. However, in a general case, quantum correlation and quantum entanglement are inequivalent, although both of them are nonnegative and invariant under the local unitary (LU) transformation $[13,14]$. For example, in the Werner state $\rho_{z}=\frac{1-z}{4} I+z|\psi\rangle\langle\psi|$ with $|\psi\rangle=(|00\rangle+|11\rangle) / \sqrt{2}$, the quantum correlation (quantum discord) [15] is greater than 0 when $z>0$, but the entanglement (concurrence) is nonzero only when $z>\frac{1}{3}$. The key difference between the two quantities is that entanglement does not increase under local operations and classical communication (LOCC) (i.e., the entanglement monotone property).

Recently, Osborne and Verstraete also proved that the distribution of bipartite entanglement among $\mathrm{N}$-qubit quantum

\footnotetext{
*zwang@hkucc.hku.hk
}

PACS number(s): 03.67.Mn, 03.65.Ud, 03.65.Ta

state satisfies the relation [16] $C_{A_{1} A_{2}}^{2}+C_{A_{1} A_{3}}^{2}+\cdots+C_{A_{1} A_{N}}^{2}$ $\leqslant \tau_{A_{1}\left(A_{2} \ldots A_{N}\right)}$, where the $\tau_{A_{1}\left(A_{2} \ldots A_{N}\right)}$ is the linear entropy for a pure state. Comparing with the three-qubit case, it is natural to ask whether or not the residual quantum correlation in an $N$-qubit pure state $(N>3)$ is a good measure of the genuine multipartite entanglement.

In this paper, we attempt to answer the above tough question clearly. Based on the quantitative complementary relations (QCRs), we analyze the properties of multipartite correlations and entanglement in four-qubit pure states. It is shown that the single residual correlation in the four-qubit case does not satisfy the entanglement monotone property. In addition, the genuine three- and four-qubit correlations are unable to quantify entanglement, either. Finally, in terms of a serious analysis on the sum of all residual correlations, we conjecture it to be an appropriate quantity for constituting the multipartite entanglement measure in the composite system.

The paper is organized as follows. In Sec. II, the properties of multipartite correlations in four-qubit pure states are analyzed in detail. As a result, a multipartite entanglement measure is conjectured. In Sec. III, we give some remarks and main conclusions. In addition, three examples are given in the Appendix.

\section{MULTIPARTITE QUANTUM CORRELATIONS IN FOUR-QUBIT PURE STATES}

Before analyzing the quantum correlations, we first introduce the QCRs. Complementarity [17] is an essential principle of quantum mechanics, which is often referred to the mutually exclusive properties of a single quantum system. As a special quantum property without classical counterpart, entanglement can constitute complementarity relations with local properties $[18,19]$. Jakob and Bergou derived a QCR for two-qubit pure state [20], i.e., $C^{2}+S_{k}^{2}=1$, in which the concurrence $C$ quantifies the nonlocal correlation of the two qubits and the $S_{k}^{2}=\left|\overrightarrow{r_{k}}\right|^{2}$ is a measure for single particle characters $\left(\overrightarrow{r_{k}}\right.$ is the polarization vector of qubit $\left.k\right)$. The experimental demonstration of this relation was made by Peng et al. [21] with nuclear magnetic resonance techniques. For an $N$-qubit pure state, the generalized QCRs are also available [21-23] 


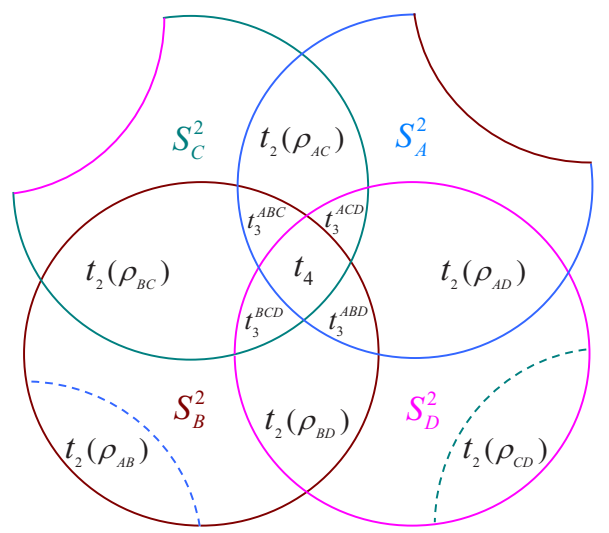

FIG. 1. (Color online) The correlation Venn diagram for a fourqubit pure state $|\Psi\rangle_{A B C D}$. The overlapping areas $t_{4}, t_{3}$, and $t_{2}$ denote the genuine four-, three-, and two-qubit quantum correlations, respectively. The areas without overlapping $S_{k}^{2}$ is the local reality of qubit $k$, for $k=A, B, C, D$.

$$
\tau_{k\left(R_{k}\right)}+S_{k}^{2}=1,
$$

where the linear entropy $\tau_{k\left(R_{k}\right)}=2\left(1-\operatorname{tr} \rho_{k}^{2}\right)[7]$ characterizes the total quantum correlation between qubit $k$ and the remaining qubits $R_{k}$.

For a two-qubit pure state, the linear entropy is a bipartite quantum correlation. For a three-qubit case, the $\tau_{k\left(R_{k}\right)}$ is composed of the two-qubit and genuine three-qubit correlations [11]. For an $\mathrm{N}$-qubit pure state [24], here we propose a natural generalization that the linear entropy is contributed by different levels of quantum correlations, i.e.,

$$
\tau_{k\left(R_{k}\right)}=t_{N}\left(|\Psi\rangle_{N}\right)+\cdots+\sum_{i<j \in R_{k}} t_{3}\left(\rho_{i j k}\right)+\sum_{l \in R_{k}} t_{2}\left(\rho_{k l}\right),
$$

where the $t_{m}$ represents the genuine $m$-qubit quantum correlation, for $m=2,3, \ldots, N$. The Venn diagram, which is often utilized in the set theory, may be employed to depict quantum correlations in a composite system. Here we draw schematically a correlation Venn diagram for a four-qubit pure state $|\Psi\rangle_{A B C D}$ in Fig. 1. Qubits $A, B, C$, and $D$ are represented by four unit circles, respectively, and the quantum correlations are denoted by the overlapping areas of these circles. According to this diagram, the four-qubit QCRs can be written as

$$
\begin{aligned}
& t_{4}+t_{3}^{(2)}+t_{3}^{(3)}+t_{3}^{(4)}+\sum_{l \in R_{A}} t_{2}\left(\rho_{A l}\right)+S_{A}^{2}=1, \\
& t_{4}+t_{3}^{(1)}+t_{3}^{(3)}+t_{3}^{(4)}+\sum_{l \in R_{B}} t_{2}\left(\rho_{B l}\right)+S_{B}^{2}=1, \\
& t_{4}+t_{3}^{(1)}+t_{3}^{(2)}+t_{3}^{(4)}+\sum_{l \in R_{C}} t_{2}\left(\rho_{C l}\right)+S_{C}^{2}=1, \\
& t_{4}+t_{3}^{(1)}+t_{3}^{(2)}+t_{3}^{(3)}+\sum_{l \in R_{D}} t_{2}\left(\rho_{D l}\right)+S_{D}^{2}=1,
\end{aligned}
$$

where the $t_{3}^{(1)}, t_{3}^{(2)}, t_{3}^{(3)}$, and $t_{3}^{(4)}$ are the three-qubit correlations in subsystems $\rho_{B C D}, \rho_{A C D}, \rho_{A B D}$, and $\rho_{A B C}$, respectively. In three-qubit pure states, the quantum correlations $t_{2}$ (square of the concurrence) and $t_{3}$ (three tangle) in the linear entropy are good measures for two- and three-qubit entanglement, respectively. However, it is an open problem that whether or not the similar relations also hold in a four-qubit pure state $|\Psi\rangle_{A B C D}$.

Before analyzing the multipartite correlations $t_{4}$ and $t_{3}^{(i)}$, we need consider how to evaluate the two-qubit correlation $t_{2}\left(\rho_{i j}\right)$ in the pure state $|\Psi\rangle_{A B C D}$. Similar to the three-qubit case, we make use of the square of the concurrence which is defined as $C_{i j}=\max \left[\left(\sqrt{\lambda_{1}}-\sqrt{\lambda_{2}}-\sqrt{\lambda_{3}}-\sqrt{\lambda_{4}}\right), 0\right]$, where the decreasing positive real numbers $\lambda_{i} \mathrm{~s}$ are the eigenvalues of matrix $\rho_{i j}\left(\sigma_{y} \otimes \sigma_{y}\right) \rho_{i j}^{*}\left(\sigma_{y} \otimes \sigma_{y}\right)$ [10]. The main reason for this evaluation is because that the relation $\Sigma_{l \in R_{k}} C_{k l}^{2}=\tau_{k\left(R_{k}\right)}$ holds for the four-qubit $W$ state $|\psi\rangle_{A B C D}=\alpha_{1}|0001\rangle+\alpha_{2}|0010\rangle$ $+\alpha_{3}|0100\rangle+\alpha_{4}|1000\rangle$ which involves only the two-qubit entanglement [11]. In the following, we will analyze the properties of the single residual correlation, the genuine threeand four-qubit correlations, and the sum of all residual correlations, respectively.

\section{A. Single residual correlation}

Under the above evaluation for the two-qubit quantum correlation, the multipartite correlation around the qubit $k$ (i.e., the residual correlation) will be

$$
M_{k}(|\Psi\rangle)=\tau_{k\left(R_{k}\right)}-\sum_{l \in R_{k}} t_{2}\left(\rho_{k l}\right)
$$

in which $t_{2}\left(\rho_{k l}\right)=C_{k l}^{2}$ and $k=A, B, C, D$. As widely accepted, a good measure for the multipartite entanglement should satisfy the following requirements [13]: (1) the quantity should be a non-negative real number; (2) it is unchanged under the LU operations; (3) it does not increase on average under the LOCC, i.e., the measure is entanglement monotone.

Now we analyze the residual correlation $M_{k}$. According to the monogamy inequality proven by Osborne and Verstraete [16], it is obvious that $M_{k}$ is positive semidefinite. In addition, for the full separable state and the entangled state involving only two-qubit correlations, it can be verified that $M_{k}=0$.

The correlation $M_{k}$ is also LU invariant, which can be deduced from the fact that the linear entropy and the concurrence are invariant under the LU transformation.

The last condition is that $M_{k}$ should be nonincreasing on average under the LOCC. It is known that any local protocol can be implemented by a sequence of two-outcome positive operator-valued measures (POVMs) involving only one party [12]. Without loss of generality, we consider the local POVM $\left\{A_{1}, A_{2}\right\}$ performed on the subsystem $A$, which satisfies $A_{1}^{\dagger} A_{1}+A_{2}^{\dagger} A_{2}=I$. According to the singular value decomposition [12], the POVM operators can be written as $A_{1}=U_{1} \operatorname{diag}\{\alpha, \beta\} V$ and $A_{2}=U_{2} \operatorname{diag}\left\{\sqrt{1-\alpha^{2}}, \sqrt{1-\beta^{2}}\right\} V$, in which $U_{i}$ and $V$ are unitary matrices. Since $M_{k}$ is LU invariant, we need only to consider the diagonal matrices in the following analysis. Note that the linear entropy and concurrence are invariant under a determinant one stochastic LOCC (SLOCC) [25], we can deduce $M_{A}\left(\left|\Phi_{1}\right\rangle\right)$ $=M_{A}\left(\frac{A_{1}|\Psi\rangle}{\sqrt{p_{1}}}\right)=\frac{\alpha^{2} \beta^{2}}{p_{1}^{2}} M_{A}(|\Psi\rangle) \quad$ and $\quad M_{A}\left(\left|\Phi_{2}\right\rangle\right)=M_{A}\left(\frac{A_{2}|\Psi\rangle}{\sqrt{p_{2}}}\right)$ 
$=\frac{\left(1-\alpha^{2}\right)\left(1-\beta^{2}\right)}{p_{2}^{2}} M_{A}(|\Psi\rangle)$, where the $p_{i}=\operatorname{tr}\left[A_{i}|\Psi\rangle\langle\Psi| A_{i}^{\dagger}\right]$ is the normalization factor. After some algebraic deductions similar to those in Refs. $[12,26]$, the following relation can be derived

$$
p_{1} M_{A}\left(\left|\Phi_{1}\right\rangle\right)+p_{2} M_{A}\left(\left|\Phi_{2}\right\rangle\right) \leqslant M_{A}(|\Psi\rangle),
$$

which means the multipartite correlation $M_{A}$ is entanglement monotone under the local operation performed on subsystem A.

It should be pointed out that the above property is not sufficient to show the parameter $M_{A}$ is monotone under the LOCC. This is because, unlike the three-qubit case, the residual correlation $M_{k}$ in a four-qubit state will change after permuting the parties. Therefore, before claiming that the $M_{k}$ is entanglement monotone, one needs to prove the parameters $M_{B}, M_{C}$, and $M_{D}$ are also nonincreasing on average under the POVM $\left\{A_{1}, A_{2}\right\}$ performed on subsystem $A$. However, this requirement cannot be satisfied in a general case, because the behaviors of the three parameters are quite different from that of $M_{A}$. For example, in the correlation $M_{C}=\tau_{C\left(R_{C}\right)}-C_{A C}^{2}-C_{B C}^{2}-C_{C D}^{2}$, only the $C_{A C}^{2}$ is invariant under the determinant one stochastic LOCC performed on subsystem A. With this property, we know $C_{A C}^{2}$ is entanglement monotone. As to the linear entropy $\tau_{C\left(R_{C}\right)}$ and the other concurrences $\left(C_{B C}^{2}\right.$ and $\left.C_{C D}^{2}\right)$, one can prove that they are decreasing and increasing under the POVM $\left\{A_{1}, A_{2}\right\}$, respectively, in terms of the following two facts: first, for the reduced density matrices $\rho_{C}, \rho_{B C}$, and $\rho_{C D}$, the effect of the POVM is equivalent to decomposing them into two mixed states, respectively; second, the linear entropy is concave function and the concurrence is convex function. Comparing the behaviors of $M_{A}$ and $M_{C}$ under the POVM, we cannot ensure that $M_{C}$ is entanglement monotone (in the Appendix, we give an example in which the correlation $M_{C}$ will increase under a selected POVM performed on subsystem $A$ ). The cases for $M_{B}$ and $M_{D}$ are similar.

For a kind of symmetric quantum state which has the property $M_{A}=M_{B}=M_{C}=M_{D}$, is the correlation $M_{k}$ entanglement monotone? The answer is still negative. Since the symmetry cannot hold after an arbitrary POVM, the parameter $M_{k}$ cannot be guaranteed to be monotone under the next level of POVM once the property is broken (see such an example in the Appendix). Therefore, we conclude that the correlation $M_{k}$ is not entanglement monotone and it is not a good entanglement measure.

\section{B. Three- and four-qubit correlations}

Next, we analyze the properties of the correlations $t_{4}$ and $t_{3}^{(i)}$. Note that the QCRs provide only four equations which cannot determine completely the five multipartite parameters in general. Therefore, a well-defined measure for $t_{3}$ or $t_{4}$ is needed in this case. Recently, an attempt was made to introduce an information measure $\xi_{1234}$ for the genuine four-qubit entanglement [24], but this measure can hardly characterize completely the genuine four-qubit correlation and/or entanglement [27].

On the other hand, a mixed three tangle $\tau_{3}$ $=\min \Sigma_{p_{x}, \phi_{x}} p_{x} \tau\left(\phi_{x}\right)[12,28]$ could not be chosen as the cor- relation $t_{3}$ either, because it is not compatible with the QCRs of Eq. (4). As an example, we consider the quantum state $|\psi\rangle_{A B C D}=(|0000\rangle+|1011\rangle+|1101\rangle+|1110\rangle) / 2 \quad$ [29], in which the reduce density matrix $\rho_{B C D}$ can be decomposed to the mix of two pure states $|\phi\rangle_{1}=|000\rangle$ and $|\phi\rangle_{2}$ $=(|011\rangle+|101\rangle+|110\rangle) / \sqrt{3}$. Supposing that the $\tau_{3}$ is a good measure for $t_{3}$, we can obtain $t_{3}^{(1)}=\tau_{3}\left(\rho_{B C D}\right)=0$ in terms of the definition of the mixed three tangle. Then the other multipartite correlations are determined from Eq. (4), with $t_{4}$ $=1.5$ and $t_{3}^{(2)}=t_{3}^{(3)}=t_{3}^{(4)}=-0.25$. Because these correlations are not in the reasonable range, the mixed three tangle is not a suitable measure compatible with the QCRs.

Although the analytical measures for $t_{4}$ and $t_{3}$ are unavailable now, we may analyze a special kind of quantum state in which $t_{4}$ is zero. The quantum state $|\varphi\rangle=\alpha|0000\rangle+\beta|0101\rangle$ $+\gamma|1000\rangle+\eta|1110\rangle$ is just the case. Suppose that the good correlation measures are existent and their values correspond to the overlapping regions in the Venn diagram (Fig. 1). It is simple to see that these correlations are non-negative and LU invariant. In the quantum state $|\varphi\rangle$, if we let the $t_{3}^{(i)}$ be the variables, we can obtain the relation $t_{3}^{(1)}=-\frac{1}{3} t_{4}$ according to the QCRs of Eq. (4). Due to the non-negative property of the two correlations, we can judge the four-qubit correlations is zero in this state. Then the other three-qubit correlations can be solved with the QCRs. In order to test the entanglement monotone of $t_{3}^{(i)}$ more clearly, the parameters in $|\varphi\rangle$ are chosen to be $\alpha=\beta=\gamma=\eta=1 / 2$ (see the example 3 in the Appendix). After performing a selected POVM, we find the $t_{3}^{(2)}$ will increase on average, which implies that the correlations $t_{3}$ and $t_{4}$ are not suitable for the quantification of entanglement.

\section{Sum of the residual correlations}

Finally, we consider the sum of all residual correlations, which is defined as

$$
M=M_{A}+M_{B}+M_{C}+M_{D}=\sum_{k} \tau_{k\left(R_{k}\right)}-2 \sum_{p>q} C_{p q}^{2},
$$

in which $k, p, q=A, B, C, D$. It is obvious that $M$ is nonnegative and LU invariant in terms of the corresponding properties of $M_{k}$. It is extremely difficult to prove the entanglement monotone property analytically. The main hindrance lies in that one cannot compare the change of the concurrences in a general quantum state before and after the POVM.

Nevertheless, we conjecture that the correlation $M$ is an entanglement monotone, as rationalized in some sense below. From the definition of $M$, it is seen that $M$ is invariant under the permutations of the subsystems. Without loss of the generality, suppose that the POVM is performed on the subsystem $A$. In this case, we analyze the behaviors of the components in $M$. According to the prior analysis in Eq. (6), the component $\xi_{1}=\tau_{A\left(R_{A}\right)}-C_{A B}^{2}-C_{A C}^{2}-C_{A D}^{2}$ is decreasing on average. Moreover, due to the concave property of linear entropy and the convex property of concurrence, the component $\xi_{2}=\tau_{B\left(R_{B}\right)}+\tau_{C\left(R_{C}\right)}+\tau_{D\left(R_{D}\right)}-2\left(C_{B C}^{2}+C_{B D}^{2}+C_{C D}^{2}\right)$ is also decreasing after the POVM. The only increasing component is $\xi_{3}=-C_{A B}^{2}-C_{A C}^{2}-C_{A D}^{2}$. It is conjectured that the decrease of 

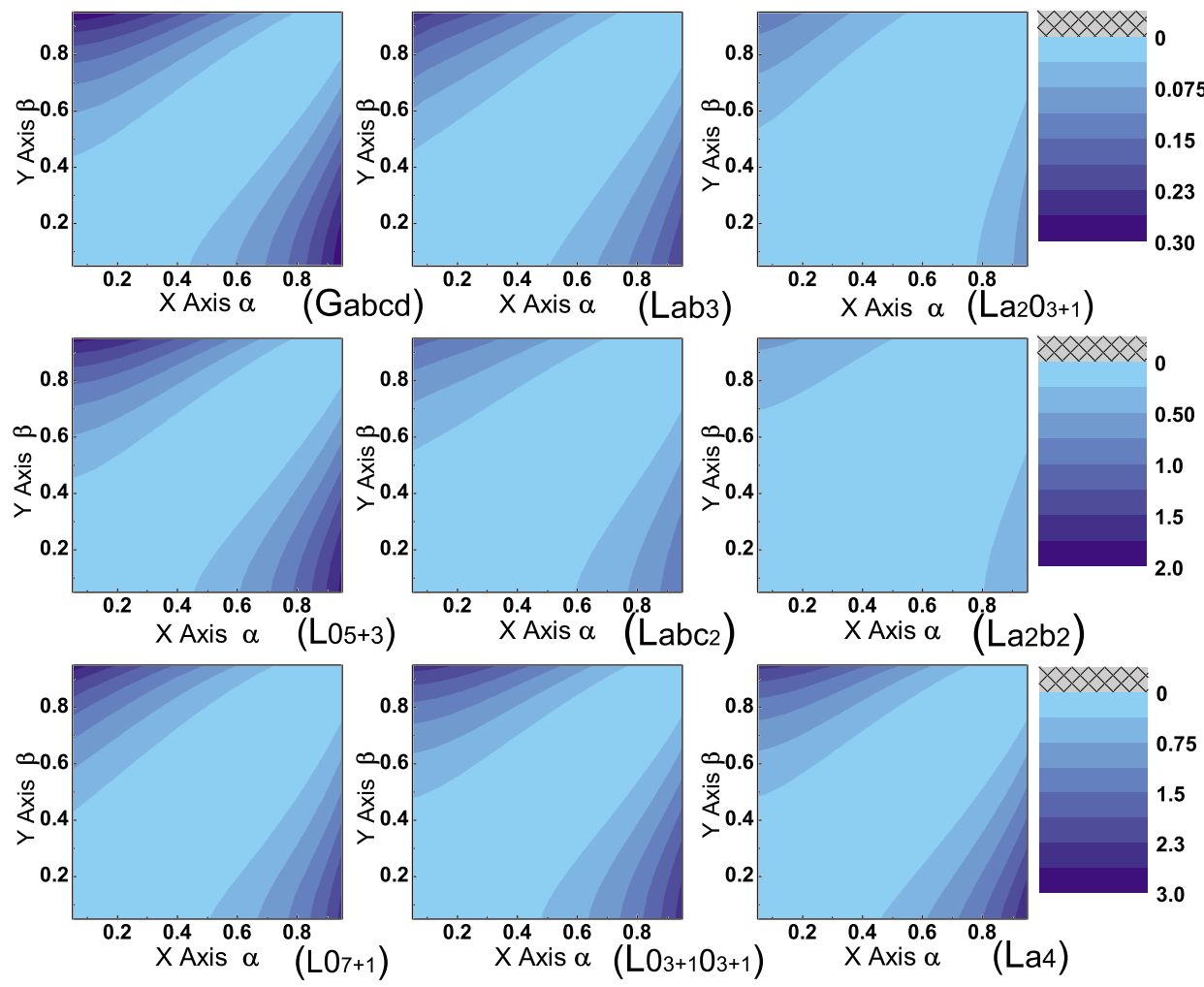

FIG. 2. (Color online) The values of $\Delta M$ for nine representative states. In the POVM, the diagonal elements $\alpha$ and $\beta$ are chosen from 0.05 to 0.95 , and the interval is 0.01 .

$\xi_{1}$ and $\xi_{2}$ can countervail the increase of $\xi_{3}$, which results further in the entanglement monotone property of $M$.

In Fig. 2, the quantity $\Delta M=M(|\Psi\rangle)-p_{1} M\left(\left|\Phi_{1}\right\rangle\right)$ $-p_{2} M\left(\left|\Phi_{2}\right\rangle\right)$ is calculated for nine quantum states $G_{a b c d}$, $L_{a b c_{2}}, L_{a_{2} b_{2}}, L_{a b_{3}}, L_{a_{4}}, L_{a_{2} 0_{3 \oplus 1}}, L_{0_{5 \oplus 3}}, L_{0_{7 \oplus 1}}$, and $L_{0_{3 \oplus 1} \overline{0}_{3 \oplus 1}}$ (the state parameters we choose are listed in Table I), which are the representative states under the SLOCC classification (cf. Ref. [29]). Due to the form of quantum state $L_{0_{3 \oplus 1} \overline{0}_{3 \oplus 1}}$ $=|0000\rangle+|0111\rangle$, we perform the POVM on its subsystem $B$.

For the other states, the POVM is performed on the subsystem $A$. From Fig. 2, we can see the correlation $M$ do not increase on average under the POVMs, which support our conjecture (for the POVMs performed on other subsystems, we obtain the similar results). In addition, for the symmetric quantum states $G_{a b c d}, L_{a b c_{2}}$, and $L_{a b_{3}}$, the second level of the POVM is also calculated and the $\Delta M$ is still non-negative (in the first level of the POVM performed on the subsystem $A$, the diagonal elements are $\alpha_{1}=0.4$ and $\beta_{1}=0.7$; in the second level of POVM, $\alpha_{2}$ and $\beta_{2}$ are chosen from 0.05 to 0.95 , and the interval is 0.01).

Mainly based on the above analysis, we therefore conjecture that the multipartite correlation $M$ is entanglement

TABLE I. The parameters we choose in the quantum states $G_{a b c d}, L_{a b c_{2}}, L_{a_{2} b_{2}}, L_{a b_{3}}, L_{a_{4}}, L_{a_{2} \mathrm{O}_{3 \oplus 1}}$ (Ref. [29]).

\begin{tabular}{lccccc}
\hline \hline$G_{a b c d}$ & $L_{a b c_{2}}$ & $L_{a_{2} b_{2}}$ & $L_{a b_{3}}$ & $L_{a_{4}}$ & $L_{a_{2} 0_{3 \oplus 1}}$ \\
\hline$a=c=1$ & $a=2$ & $a=1$ & $a=1$ & $a=1$ & $a=1$ \\
$b=d=0.5$ & $b=c=1$ & $b=1$ & $b=1.5$ & & \\
\hline \hline
\end{tabular}

monotone and then is possible to constitute a measure for the total multipartite entanglement in four-qubit pure states.

At this stage, we may also introduce the average multipartite entanglement

$$
E_{m s}=\frac{M}{4}=\frac{M_{A}+M_{B}+M_{C}+M_{D}}{4},
$$

to characterize the entanglement per single qubit (ranged in $[0,1]$ ), as far as the correlation $M$ is (conjectured to be) entanglement monotone. A remarkable merit of this quantity is its computability. For the quantum state $\quad G_{a b c d}=\frac{a+d}{2}(|0000\rangle+|1111\rangle)+\frac{a-d}{2}(|0011\rangle+|1100\rangle)$ $+\frac{b+c}{2}(|0101\rangle+|1010\rangle)+\frac{b-c}{2}(|0110\rangle+|1001\rangle)$ which is the generic kind under the SLOCC classification, the change of $E_{m s}$ with the real parameters $a$ and $d$ are plotted in Fig. 3 (the parameters $b=0$ and $c=0.5$ are fixed). In the regions near $(a=d=0),(a \gg c, d)$, and $(d \gg a, c)$, the multipartite entanglement $E_{m s}$ tends to zero, which can be explained that the quantum state tends to be the tensor product of the two bell states in these ranges. The bigger values of $E_{m s}$ appear in the regions near $(a=0, d=0.5),(a=0.5$ and $d=0)$, and $a=d \gg c$. This is because the quantum state $G_{a b c d}$ approaches to the four-qubit $G H Z$ state in these regions [e.g., when $a=0$ and $d=0.5$, the $E_{m s}$ is 1 and the quantum state can be rewritten as $G_{a b c d}=(|\alpha \alpha \alpha \alpha\rangle+|\beta \beta \beta \beta\rangle) / \sqrt{2}$ after a local unitary transformation $|\alpha\rangle=(|0\rangle+i|1\rangle) / \sqrt{2}$ and $|\beta\rangle=(|0\rangle-i|1\rangle) / \sqrt{2}]$. In this case, the four-partite entanglement is a dominant one.

Although the operational meaning of $E_{m s}$ for entanglement transformation and distillation is not clear now, we can 


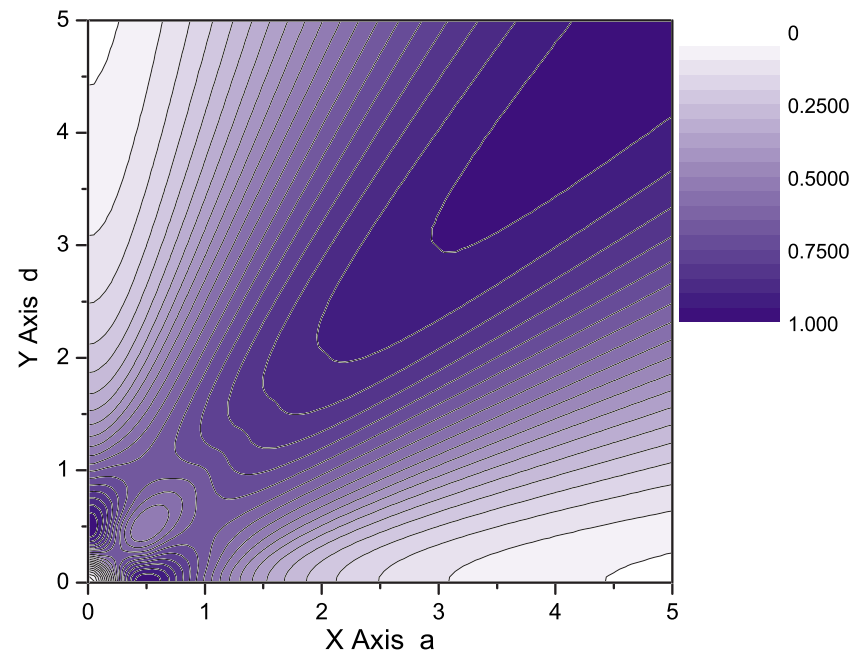

FIG. 3. (Color online) The average multipartite entanglement $E_{m s}$ for the quantum state $G_{a b c d}$ in which the parameters $a$ and $d$ are chosen from 0 to 5 and the interval is 0.05 . The parameters $b=0$ and $c=0.5$ are fixed.

use this quantity to restrict some procedures which are impossible (suppose that the $E_{m s}$ is validated to be entanglement monotone). For example, if the quantity increases in an LOCC transformation from $\left|\varphi_{1}\right\rangle$ to $\left|\varphi_{2}\right\rangle$, we can judge that this procedure is impossible because the entanglement should be monotone in a real physical transformation.

It should be pointed out that the quantity $E_{m s}$ in Eq. (8) corresponds to the correlation $t_{4}+\frac{3}{4} \sum t_{3}^{(i)}$, which is not the total multipartite correlation $M_{T}=t_{4}+\Sigma t_{3}^{(i)}$ in the Venn diagram. Whether or not the $M_{T}$ is a good candidate for the total multipartite entanglement in the system is worth study in the future. In order to test the entanglement properties of $M_{T}$, one needs first to find the appropriate definitions for the correlation $t_{4}$ and $t_{3}$, respectively.

For an $N$-qubit pure state, the sum of all residual correlations is given by

$$
\begin{aligned}
M_{N}\left(\Psi_{N}\right) & =N t_{N}+(N-1) \sum t_{N-1}+\cdots+3 \sum t_{3} \\
& =\sum \tau_{k\left(R_{k}\right)}-2 \sum \sum_{i>j} C_{i j}^{2} .
\end{aligned}
$$

Similar to the four-qubit case, this quantity is non-negative real number in terms of the monogamy inequality. In addition, the LU invariance of $M_{N}$ is guaranteed by the corresponding property of linear entropy and concurrence. For the entanglement monotone, we conjecture the correlation $M_{N}$ also satisfies. Therefore, correlation $M_{N}$ may be able to characterize the multipartite entanglement in the system. Similarly, the average over $N$ qubits $M_{N} / N$ (ranged in $[0,1]$ ) can be considered as the entanglement per qubit.

\section{DISCUSSION AND CONCLUSION}

In the correlation Venn diagram of three-qubit pure state $|\Psi\rangle_{A B C}[23,30]$, the quantum correlations at different levels are able to characterize the corresponding quantum entangle-
TABLE II. The values of the correlation measures related to subsystem $C$ before and after the POVM.

\begin{tabular}{lccccc}
\hline \hline State Correlation & $\tau_{C\left(R_{C}\right)}$ & $C_{A C}^{2}$ & $C_{B C}^{2}$ & $C_{C D}^{2}$ & $M_{C}$ \\
\hline$|\Psi\rangle$ & $8 / 9$ & $4 / 9$ & 0 & 0 & $4 / 9$ \\
$\left|\Phi_{1}\right\rangle$ & 0.9994 & 0.04703 & 0 & 0 & 0.9524 \\
$\left|\Phi_{2}\right\rangle$ & 0.4867 & 0.4063 & 0 & 0 & 0.08042 \\
\hline \hline
\end{tabular}

ments. Therefore, the total entanglement in the system is contributed by the two-qubit entanglement and the genuine three-qubit entanglement, respectively. However, in the fourqubit case, the structure of total entanglement is quite complicated; how to quantify separately the three- and four-qubit entanglement is still an open problem. It was indicated by $\mathrm{Wu}$ and Zhang that the set of two-, three-, four-partite Greenberger-Horne-Zeilinger (GHZ) states is not a reversible entanglement generating set for four-party pure states [31] (i.e., the set of entangled states cannot generate an arbitrary four-party pure state by the LOCC asymptotically [32]), which implies that the GHZ-class entanglements are not sufficient for characterizing the structure of total entanglement in the system. Recently, it was noted by Lohmayer et al. [33] that a kind of rank-two three-qubit mixed states which are entangled but do not have the mixed three tangle and concurrence (one can consider that these states are reduced from four-qubit pure states). This case shows further that the quantification of entanglement in multiqubit systems is extremely complicated and highly nontrivial.

In conclusion, based on the generalized QCRs, we have analyzed the multipartite correlations in four-qubit pure states. Unlike the three-qubit case, we find that the similar relations do not hold again in the four-qubit system. First, the residual correlation $M_{k}$ is not of entanglement monotone. In addition, the genuine three- and four-qubit correlations are not suitable to be entanglement measure, either. Finally, the total residual correlation $M$ has been analyzed, and it is conjectured that the average multipartite correlation $E_{m s}$ is able to quantify the multipartite entanglement in the system.

\section{ACKNOWLEDGMENTS}

The work was supported by the RGC of Hong Kong under Grants No. HKU 7051/06P, No. 7012/06P, and No. HKU $3 / 05 \mathrm{C}$, the URC fund of HKU, NSF-China under Grant No. 10429401 .

\section{APPENDIX}

\section{Example 1}

Consider a quantum state $|\Psi\rangle_{A B C D}=(|0000\rangle+|0011\rangle$ $+|0101\rangle+|0110\rangle+|1010\rangle+|1111\rangle) / \sqrt{6}$, which belongs to the representative state $L_{a_{2} b_{2}}$ (the parameters is chosen as $a=b=1)$ under the SLOCC classification [29]. The POVM $\left\{A_{1}, A_{2}\right\}$ is performed on subsystem $A$, which has the form 
TABLE III. The values of the correlation measures related to subsystem before and after the second level of the POVM.

\begin{tabular}{lccccc}
\hline \hline State & & & & \\
\hline & $\tau_{A\left(R_{A}\right)}$ & $C_{A B}^{2}$ & $C_{A C}^{2}$ & $C_{A D}^{2}$ & $M_{A}$ \\
\hline$\left.\Phi_{1}\right\rangle$ & 0.4324 & 0 & 0.2767 & 0 & 0.1556 \\
$\left|\Phi_{11}\right\rangle$ & 0.9960 & 0 & 0.2408 & 0 & 0.7552 \\
$\left|\Phi_{12}\right\rangle$ & 0.1565 & 0 & 0.07749 & 0 & 0.07901 \\
\hline \hline
\end{tabular}

$A_{1}=U_{1} \operatorname{diag}\{\alpha, \beta\} V$ and $A_{2}=U_{2} \operatorname{diag}\left\{\sqrt{1-\alpha^{2}}, \sqrt{1-\beta^{2}}\right\} V$. Due to the LU invariance of the correlation $M_{k}$, we need only to consider the diagonal matrices in which the parameters are chosen to be $\alpha=0.9$ and $\beta=0.2$. After the POVM, two outcomes $\left|\Phi_{1}\right\rangle=A_{1}|\Psi\rangle / \sqrt{p_{1}}$ and $\left|\Phi_{2}\right\rangle=A_{2}|\Psi\rangle / \sqrt{p_{2}}$ are present, with the possibilities as $p_{1}=0.5533$ and $p_{2}=0.4467$. Some calculated results are listed in Table II.

According to these values, we can deduce that $M_{C}(|\Psi\rangle)-\left[p_{1} M_{C}\left(\left|\Phi_{1}\right\rangle\right)+p_{2} M_{C}\left(\left|\Phi_{2}\right\rangle\right)\right]=-0.1185, \quad$ which means that the correlation $M_{C}$ is increasing under the LOCC.

\section{Example 2}

Consider a symmetric quantum state $\quad|\Psi\rangle=(3|0000\rangle+3|1111\rangle-|0011\rangle-|1100\rangle+3|0101\rangle$ $+3|1010\rangle+|0110\rangle+|1001\rangle) / 2 \sqrt{10}$, which belongs to the representative state $G_{a b c d}$ (the state parameters are chosen as $a$ $=c=0.5$ and $b=d=1$ ) [29]. According to the analysis in Sec. II A, we know that the correlation $M_{k}$ is monotone under the first level of the POVM. In this example, we will show that the correlation $M_{A}$ will be increasing under the second level of the POVM.

The first level of POVM $\left\{A_{1}, A_{2}\right\}$ is performed on the subsystem $A$ in which the diagonal elements are $\alpha=0.3$ and $\beta=0.8$. After the POVM, two outcomes $\left|\Phi_{1}\right\rangle$ and $\left|\Phi_{2}\right\rangle$ can be obtained with the probabilities $p_{1}=0.3650$ and $p_{2}=0.6350$, respectively. Suppose that $|\Phi\rangle_{1}$ is gained. Then we do the second level of POVM $\left\{A_{11}, A_{12}\right\}$ on the subsystem $C$, in
TABLE IV. The values of the correlation measures $t_{4}$ and $t_{3}$ before and after the POVM.

\begin{tabular}{lccccc}
\hline \hline State Correlation & & $t_{4}^{(1)}$ & $t_{3}^{(2)}$ & $t_{3}^{(3)}$ & $t_{3}^{(4)}$ \\
\hline$|\Psi\rangle$ & 0 & 0 & 0.2500 & 0.2500 & 0.2500 \\
$\left|\Phi_{1}\right\rangle$ & 0 & 0 & 0.02721 & 0.1377 & 0.1377 \\
$\left|\Phi_{2}\right\rangle$ & 0 & 0 & 0.6651 & 0.1504 & 0.1504 \\
\hline \hline
\end{tabular}

which the diagonal elements are chosen to be $\alpha_{1}=0.9$ and $\beta_{1}=0.2$. The outcomes $\left|\Phi_{11}\right\rangle$ and $\left|\Phi_{12}\right\rangle$ are obtained with the probabilities $p_{11}=0.1929$ and $p_{12}=0.8071$, respectively. The calculated results are presented in Table III.

Comparing the change of $M_{A}$, we can obtain $M_{A}\left(|\Phi\rangle_{1}\right)-\left[p_{11} M_{A}\left(\left|\Phi_{11}\right\rangle\right)+p_{12} M_{A}\left(\left|\Phi_{12}\right\rangle\right)\right]=-0.05382 \quad$ This means that the correlation $M_{A}$ is increasing under the LOCC, and thus $M_{k}$ is not a good entanglement measure for the symmetric quantum state.

\section{Example 3}

We analyze the quantum state $|\Psi\rangle_{A B C D}=(|0000\rangle+|0101\rangle$ $+|1000\rangle+|1110\rangle) / 2$, which is the representative state $L_{0_{5 \oplus 3}}$ [29]. The POVM $\left\{A_{1}, A_{2}\right\}$ is performed on the subsystem $B$. Due to the LU invariance of the correlations $t_{4}$ and $t_{3}$, we only consider the diagonal elements of the operators $A_{1}$ and $A_{2}$ (in the form of the singular value decomposition) in which the parameters are chosen to be $\alpha=0.9$ and $\beta=0.4$. After the POVM, two outcomes $\left|\Phi_{1}\right\rangle$ and $\left|\Phi_{2}\right\rangle$ are obtained with the probabilities $p_{1}=0.4850$ and $p_{2}=0.5150$, respectively. In Table IV, the values of $t_{4}$ and $t_{3}^{(i)}$ for $|\Psi\rangle,\left|\Phi_{1}\right\rangle$ and $\left|\Phi_{2}\right\rangle$ are listed.

With these values, we can obtain $t_{3}^{(2)}(|\Psi\rangle)-\left[p_{1} t_{3}^{(2)}\left(\left|\Phi_{1}\right\rangle\right)\right.$ $\left.+p_{2} t_{3}^{(2)}\left(\left|\Phi_{2}\right\rangle\right)\right]=-0.1057$, which means that the correlation $t_{3}$ can increase on average under the LOCC and that it is not a good entanglement measure.
[1] A. K. Ekert, Phys. Rev. Lett. 67, 661 (1991).

[2] C. H. Bennett, G. Brassard, C. Crépeau, R. Jozsa, A. Peres, and W. K. Wootters, Phys. Rev. Lett. 70, 1895 (1993).

[3] C. H. Bennett and D. P. Divincenzo, Nature (London) 404, 247 (2000).

[4] R. Raussendorf and H. J. Briegel, Phys. Rev. Lett. 86, 5188 (2001).

[5] S.-S. Li, G.-L. Long, F.-S. Bai, S.-L. Feng, and H.-Z. Zheng, Proc. Natl. Acad. Sci. U.S.A. 98(21), 11847 (2001).

[6] C. H. Bennett, D. P. DiVincenzo, J. A. Smolin, and W. K. Wootters, Phys. Rev. A 54, 3824 (1996).

[7] E. Santos and M. Ferrero, Phys. Rev. A 62, 024101 (2000).

[8] G. Vidal and R. F. Werner, Phys. Rev. A 65, 032314 (2002).

[9] M. B. Plenio, S. Virmani, Quantum Inf. Comput. 7, 1 (2007).

[10] W. K. Wootters, Quantum Inf. Comput. 1, 27 (2001); S. Hill and W. K. Wootters, Phys. Rev. Lett. 78, 5022 (1997); W. K. Wootters, ibid. 80, 2245 (1998).

[11] V. Coffman, J. Kundu, and W. K. Wootters, Phys. Rev. A 61, 052306 (2000).

[12] W. Dür, G. Vidal, and J. I. Cirac, Phys. Rev. A 62, 062314 (2000).

[13] V. Vedral, M. B. Plenio, M. A. Rippin, and P. L. Knight, Phys. Rev. Lett. 78, 2275 (1997).

[14] D. L. Zhou, B. Zeng, Z. Xu, and L. You, Phys. Rev. A 74, 052110 (2006); L. Henderson and V. Verdral, J. Phys. A 34, 6899 (2001).

[15] H. Ollivier and W. H. Zurek, Phys. Rev. Lett. 88, 017901 (2001).

[16] T. J. Osborne and F. Verstraete, Phys. Rev. Lett. 96, 220503 (2006). 
[17] N. Bohr, Nature (London) 121, 580 (1928).

[18] S. Bose and D. Home, Phys. Rev. Lett. 88, 050401 (2002).

[19] J. Oppenheim, K. Horodecki, M. Horodecki, P. Horodecki, and R. Horodecki, Phys. Rev. A 68, 022307 (2003).

[20] M. Jakob and J. A. Bergou, e-print arXiv:quant-ph/0302075.

[21] X. Peng, X. Zhu, D. Suter, J. Du, M. Liu, and K. Gao, Phys. Rev. A 72, 052109 (2005).

[22] T. E. Tessier, Found. Phys. Lett. 18, 107 (2005).

[23] B. Chong, J. Du, X. Peng, and H. Keiter (private communication).

[24] J.-M. Cai, Z.-W. Zhou, X.-X. Zhou, and G.-C. Guo, Phys. Rev. A 74, 042338 (2006).

[25] F. Verstraete, J. Dehaene, and B. De Moor, Phys. Rev. A 68, 012103 (2003).

[26] A. Wong and N. Christensen, Phys. Rev. A 63, 044301 (2001).
[27] For instance, when the parameter $a$ is chosen to be 100 (or to approach the infinite), the quantum state $|\phi\rangle=a(|0000\rangle$ $+|0101\rangle+|1010\rangle+|1111\rangle)+i|0001\rangle+|0110\rangle-i|1011\rangle[29]$ tends to be the tensor product of two Bell states, while the measure $\xi_{1234}$ is about 0.99985 (approaches to 1) in this state.

[28] A. Uhlmann, Phys. Rev. A 62, 032307 (2000).

[29] F. Verstraete, J. Dehaene, B. De Moor, and H. Verschelde, Phys. Rev. A 65, 052112 (2002).

[30] J.-M. Cai, Z.-W. Zhou, and G.-C. Guo, Phys. Lett. A 363, 392 (2007).

[31] S. Wu and Y. Zhang, Phys. Rev. A 63, 012308 (2000).

[32] C. H. Bennett, S. Popescu, D. Rohrlich, J. A. Smolin, and A. V. Thapliyal, Phys. Rev. A 63, 012307 (2000).

[33] R. Lohmayer, A. Osterloh, J. Siewert, and A. Uhlmann, Phys. Rev. Lett. 97, 260502 (2006). 